\title{
Prediction and Disarming of Drain Sink Formation during Unsteady-state Bottom Teeming
}

\author{
Niklas KOJOLA, ${ }^{1)}$ Shigeo TAKAGI, ${ }^{2)}$ Shinichiro YOKOYA ${ }^{1)}$ and Pär JÖNSSON ${ }^{1)}$ \\ 1) Division of Applied Process Metallurgy, Royal Institute of Technology, SE-100 44 Stockholm, Sweden. \\ 2) Department of Mechanical Engineering, Nippon Institute of Technology, Miyashiromachi, Saitama 345-8501 Japan.
}

(Received on July 15, 2008; accepted on October 23, 2008)

\begin{abstract}
The behavior of an unsteady-state drain sink as function of outlet diameter, outlet length, supernatant phase viscosity, supernatant phase density and supernatant phase layer thickness was studied using physical modeling. Two new hydrodynamic models for the prediction of the drain sink formation height were derived and compared to models found in literature. Both could accurately predict the drain sink formation height in their respective domain as function of all variables mentioned above, except supernatant phase viscosity since its influence on drain sink formation height was negligible in the experimental systems. Finally, the influence of the vessel bottom shape on the increased yield of a steel plant teeming operation is discussed.
\end{abstract}

KEY WORDS: drain sink; non-vortexing funnel; outlet size; outlet length; slag density; slag viscosity and bottom shape.

\section{Introduction}

Slag carry-over from ladle to tundish and tundish to mould during continuous casting may cause slag emulsification, reoxidation and dissolution of other unwanted substances into the steel melt. ${ }^{1)}$ The inclusions, which may be formed during the slag entrainment, are difficult to remove since they appear immediately before the melt enters the mould. For example, some of the defects in automobile sheets have been found to origin from emulsified slag. ${ }^{2}$

Since a slag usually has lower density than the steel melt, it floats on top of the melt. However, when the bath level is low enough, the maximum flow through the outlet becomes larger than the maximum flow to the outlet. This gives that a part of the outlet cross-section area will be penetrated by a second phase, which, depending on the situation, may be either the gas in the atmosphere or a supernatant phase, for example a slag (from now on, the supernatant phase is referred to as second phase). This phenomenon is a called drain sink or a non-vortexing funnel. It determines the lowest level to which the vessel can be teemed without slag entrainment. On one hand, every centimeter of lowered bath level in a $2 \mathrm{~m}$ diameter ladle increases the yield with almost one ton of steel while on the other hand, slag carry-over decreases the product quality. Thus, it would be beneficial both to be able to exactly predict the drain sink formation height and to be able to minimize the steel left in the ladle when the drain sink forms. By physical modeling, the present work investigates the drain sink as function of second phase density, viscosity and layer thickness. In the first part of the paper, the previous theories predicting the drain sink phenomena as well as the experimental conditions of the current study are given. Thereafter, the physical modeling results are presented and discussed. Finally, the development of two new models which predict the drain sink formation height in presence of second phase, are presented.

\section{Previous Studies}

The drain sink is a funnel shaped radius directed openchannel teeming flow where the horizontal bulk flow towards an outlet is smaller than the maximum possible flow through the outlet, ${ }^{3)}$ like a waterfall. In steel practice, the drain sink height (i.e. the critical height where the drain sink forms) sets the lower limit of the bath level at which the teeming should be terminated in order to avoid slag carry-over. Thus, the drain sink phenomenon has a direct influence on the production yield of a steel industry teeming process. In contrast to the visually, but not physically, similar vortex, the drain sink always forms independent of the initial velocity conditions in the vessel being teemed. Furthermore, it has also been suggested that drain sink is the only of the two phenomena that occur during steel making. ${ }^{4)}$

In order to understand the drain sink, some simple calculations based on hydrodynamic principles can be done leading to Eq. (1):

$$
\lim _{H \rightarrow 0} \frac{\pi \cdot R^{2} S \sqrt{H}}{2 \pi \cdot r H T \sqrt{H}}=\lim _{H \rightarrow 0} \frac{R}{2 H} U=\infty
$$

where $r$ is the outlet radius and $H$ is liquid bath height. Furthermore, $S, T$ and $U$ are constants that can be determined experimentally. From Eq. (1), it can be seen that at some 
critical bath level height the volume flow rate towards the outlet (the denominator of Eq. (1)) will be smaller than the flow through the outlet (the numerator in Eq. (1)), which will lead to the formation of a two-phase funnel shaped interface through the liquid.

The drain sink has been analyzed thoroughly by many authors ${ }^{1,3-8)}$ and some details about previous publications can be found in Table 1. Andrzejewski et al. and Mazzaferro et al. claims that the drain sink is the main slag transporting phenomena. ${ }^{1,4)}$ Andrzewjeski et al. reports that onset of drain sink flow is indicated by a hydraulic jump, signifying that the flow velocity has become critical. ${ }^{1)}$ A hydrodynamic critical-condition-model has been presented by Lubin and Springer, where the critical drain sink height, $H_{\text {cr }}$, can be expressed as follows ${ }^{11)}$ :

$$
H_{\mathrm{cr}}=\left(\frac{1}{2 \pi^{2}} \cdot \frac{Q_{\mathrm{cr}}^{2}}{g\left(1-\frac{\rho_{2}}{\rho_{1}}\right)}\right)
$$

where $Q_{\text {cr }}$ is the critical volumetric outflow rate, $H_{\mathrm{cr}}$ is the liquid bath height and $\rho_{1}$ and $\rho_{2}$ is density of primary phase 1 and supernatant phase 2. This relationship has been slightly modified (for example multiplied with an empirical constant) by other authors and is reported to give good agreement when comparing the predictions to experimental data. $^{3)}$

Another critical condition for drain sink formation has been developed by Andrzejewski et al. ${ }^{1)}$ based on a flow rate comparison. It utilizes cylindrical control volume and is based on the statement that the critical velocity is the velocity when the Froude number equals 1. It does not consider the effect of second phase density on the drain sink phenomenon.

The drain sink height is reported to be independent of bottom nozzle position, ${ }^{1,3)}$ initial filling height, vessel diameter and the shape of the vessel bottom (flat, hemispherical, sloped or inclined), but is reported to be a linear function of outlet diameter ${ }^{1,3,5)}$ although there are different opinions regarding the proportionality constant. The drain sink radius is reported to increase in presence of an olive oil cover. ${ }^{8)}$

One solution to the drain sink problem is to decrease the outlet area during late stages of teeming since that reduces the flow rate and then the flow velocities. Partial nozzle closing has been tried experimentally with a lowered drain sink height as result. ${ }^{3)}$ However, it is not a desirable solution during production since a rapid ladle change is necessary to avoid problems related to low bath level in the tundish (insufficient inclusion removal, slag emulsification, temperature drop, slag carry-over to the mould etc.). Another method that has been pointed out is an inclination of the ladle bottom which reduces the amount of steel left when the drain sink forms and thus increases the exchange factor of the process. ${ }^{49)}$ There also exist different slag detection systems operating in the outlet region but the main purpose of these are to detect rather than prevent the drain sink flow. ${ }^{10)}$

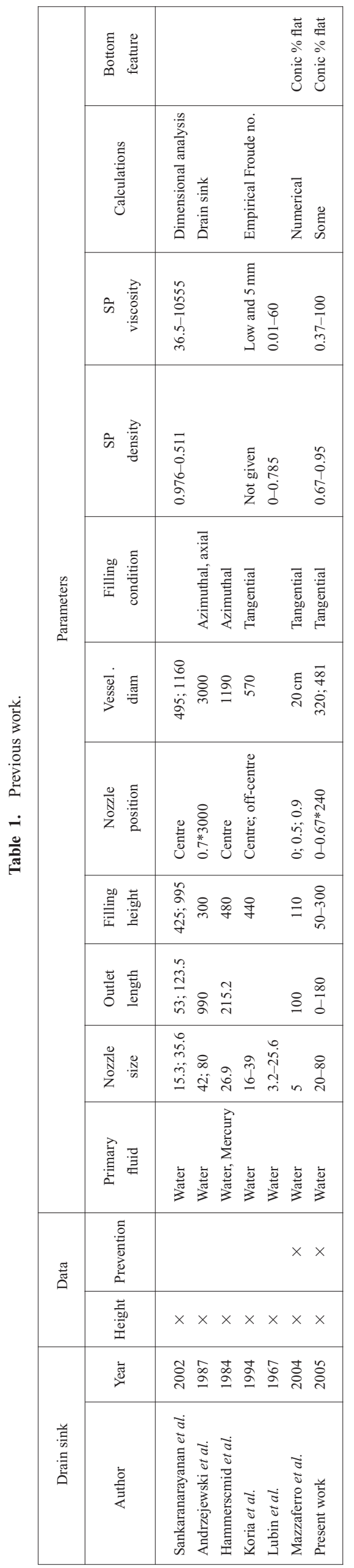




\section{Experimental Work}

Two circular and transparent vessels with changeable bottom plates and inner diameters of $320 \mathrm{~mm}$ and $480 \mathrm{~mm}$ respectively were made from acrylic resin. In the smaller vessel, various circular bottom plates equipped with different circular center placed outlets could be used. The diameters of the outlets were $20,40,60$ and $80 \mathrm{~mm}$. In addition, the $60 \mathrm{~mm}$ diameter outlet could be fitted with outlet nozzles with lengths ranging from 0 to $180 \mathrm{~mm}$. There were also coned bottoms available with $160 \mathrm{~mm}$ base diameter and $20 \mathrm{~mm}$ outlet diameter and various slope angels (20, $30,40,50,60,70$ degrees). The larger vessel had a similar set-up, but the outlet could be placed with either $0,50,90$, 130 or $160 \mathrm{~mm}$ radial distance to the center. For details, see Fig. 1.

In the physical model, water was used as the primary liquid to simulate steel. This is because water's kinematic viscosity at $25^{\circ} \mathrm{C}$ is $1 \times 10^{-6} \mathrm{~m}^{2} / \mathrm{s}^{13)}$ which is very similar to the kinematic viscosity of liquid steel which at $1600^{\circ} \mathrm{C}$ is $0.8 \times 10^{-6} \mathrm{~m}^{2} / \mathrm{s} .{ }^{14)}$ The supernatant slag present in steel making was modeled by silicon oils and $n$-pentane. These does not in any way represent perfect fluids to model slag, since slags are complex structures where small deviances regarding composition and temperature can result in great changes of viscosity and aggregation state. ${ }^{14,15)}$ It has also been reported that, in order to represent reality, the viscosity and density ratios between the two phases of the cold model should be similar to the corresponding ratios between actual steel and slag. ${ }^{16)}$ Industrial slag/steel viscosity ratios usually range from 50 to 1500 while industrial slag/steel densities usually are around $0.35 .^{3)}$ Unfortunately, fluids that fulfill the viscosity ratio condition usually exceed the density ratio condition. Thus, the present work does not pretend to represent a perfect model of steel and slag, but to

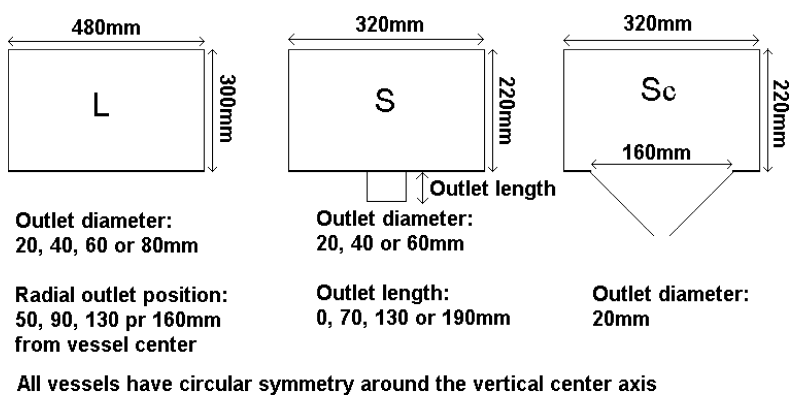

Fig. 1. Experimental vessel L, S and Sc (S with coned bottom) used in the experiments. qualitatively analyze relations among the quantities. The materials (and corresponding properties) used as second phases can be seen in Table 2. Surface energies are not taken into consideration, but the interfacial energy difference is small between the interfaces silicon oil/water, $n$ pentane/water and air/water as seen in Table 2. All experiments were recorded using a digital video camera placed at outlet level, $4 \mathrm{~m}$ from the experimental vessel which gives an error less than $5 \%$ of the measured value.

\section{Theory}

\subsection{Existing Drain Sink Models}

The Lubin and Springer hydrodynamic drain sink model $^{11)}$ is a flow balance relation between the bath height and the radius of a half sphere control volume regarding volume flow. The flow though the half sphere control volume surrounding the outlet should equal the flow through the outlet. It is assumed that the flow velocity is normal to the surface of the half sphere and equals the velocity given by the Bernoulli equation in a point at the top of the sphere, which is at a height equal to the sphere radius. The pressure at this point is assumed to equal the static pressure created by a low density second phase layer with known thickness, resting on top of the primary phase and locally stretching down to the top of the sphere. Since the flow through the sphere equals the product of the velocity at its top and its surface area, it is zero when the radius is zero and when the radius equals the bath height and positive in-between. Since the outlet area is constant while the maximum area of the half sphere is determined by the bath height and since the bath height can be very small, there is a limiting bath height below which no solution for the sphere radius exists. The Lubin and Springer model defines this critical bath height as the drain sink height and finds it by setting $d H / d r=0$, where $H$ is bath level height and $r$ is sphere radius. It is obvious that the constant velocity assumed to be distributed over the sphere is lower than predicted by the Bernoulli equation and in addition, the flow towards the outlet cannot be spherical when the bath level is smaller than the outlet radius.

There is also a model based on similar principles but with cylindrical control volume, here called the Andrzejewski model. ${ }^{1)}$ It assumes all flow towards the outlet to be directed normal to a cylinder surrounding it and defines the critical radial velocity as the velocity when the Froude number equals 1 . It does not consider effect of second phase.

Table 2. Data for the experimental fluids.

\begin{tabular}{|c|c|c|c|c|c|c|}
\hline Trading name & Manufacturer & Name & $\begin{array}{l}\text { Density } \\
\left(\mathrm{kg} / \mathrm{dm}^{3}\right)\end{array}$ & $\begin{array}{c}\text { Viscosity } \\
\left(\mathrm{mm}^{2} / \mathrm{s}, \mathrm{cSt}\right)\end{array}$ & $\begin{array}{l}\text { Interfacial energy } \\
\text { (air) }(\mathrm{mN} / \mathrm{m})\end{array}$ & $\begin{array}{c}\text { Interfacial energy } \\
\text { (water) }(\mathrm{mN} / \mathrm{m})\end{array}$ \\
\hline Silicon oil $100^{17)}$ & Shin Etsu & KF96-100cs & 0.965 & 100 & 20.9 & 53 \\
\hline Silicon oil $20^{18)}$ & Rhodorsil & $47 \mathrm{~V} 20 \mathrm{R}$ & 0.95 & 20 & - & - \\
\hline Silicon oil $10^{17)}$ & Shin Etsu & KF96-10cs & 0.935 & 10 & 20.1 & 52.7 \\
\hline$n$-Pentane $\mathrm{e}^{19)}$ & JUNSEI & $5 \mathrm{~F} 2181$ & 0.626 & 0.37 & - & 57.8 \\
\hline Water $^{20)}$ & - & - & 0.998 & 1 & 72.8 & - \\
\hline
\end{tabular}




\subsection{New Drain Sink Models}

The nature of the drain sink is simple, the maximum flow to the outlet becomes smaller than the flow through the outlet. The complexity arises on how to calculate the volume flow to the outlet and how to treat the effect of a second phase. Below, two new hydrodynamic drain sink models are derived, one with spherical control volume (called NMs) and one with cylindrical control volume (called NMc). Both take in to consideration the second phase density and layer thickness. Furthermore, both use the Bernoulli equation-based velocity distributions over the control volume. In addition, both models are based on the critical flow balance equation seen in the following equation:

$$
Q_{\mathrm{TH}}\left(H, l, g, t, \rho_{1}, \rho_{2}, R\right)=Q_{\mathrm{TO}}\left(H, l, g, t, \rho_{1}, \rho_{2}, A\right) .
$$

where $Q_{\mathrm{TO}}$ is maximum flow to the outlet, $Q_{\mathrm{TH}}$ is maximum flow through the outlet, $H$ is bath level height, $l$ is outlet length, $T$ is second phase layer thickness, $\rho_{1}$ is primary phase density, $\rho_{2}$ is second phase density, $R$ is outlet radius and $A$ is surface area of the control volume surrounding the outlet. The definition of maximum flow is here that the static fluid pressure is atmospheric at the outlet (where the fluid leaves the system) and is determined by the static pressure of the second phase at rest on the surface of the control volume. The flow velocities are assumed to be normal to the surface area of the control volume and the spherical, cylindrical and outlet control volumes can all be seen in Fig. 2.

The Bernoulli equation for point $\mathrm{C}$ and $\mathrm{B}$ on a stream line can be written as follows:

$$
P_{\mathrm{C}}+\rho_{1} g h_{\mathrm{C}}+\frac{\rho_{1} v_{\mathrm{C}}^{2}}{2}=P_{\mathrm{B}}+\rho_{1} g h_{\mathrm{B}}+\frac{\rho_{1} v_{\mathrm{B}}^{2}}{2}
$$

where $P$ is pressure, $h$ is height, $v$ is velocity and index 1 indicates the primary phase. If $\mathrm{C}$ is a point at the interface between primary phase 1 and supernatant phase 2 and far from the outlet, where the liquids are at rest, the pressure and height at point $\mathrm{C}$ can be calculated as follows:

$$
\begin{gathered}
P_{\mathrm{C}}=P^{\circ}+t \rho_{2} g . . \\
h_{\mathrm{C}}=H \ldots \ldots . . .
\end{gathered}
$$

where $P^{\circ}$ is the surrounding pressure and index 2 indicates the supernatant phase and $t$ is the supernatant phase layer thickness. The velocity at point $\mathrm{C}$ can be approximated to zero as shown in Eq. (7). This is because the movement of the two phase interface where $\mathrm{C}$ is situated is very slow compared to the fluid velocity close to the outlet which in case is caused by the mass balance which, for incompressible fluids, yields that the flow rate through the relatively narrow outlet cross-sectional area must equal the product of the vertical velocity of the bath surface area and the relatively large cross-sectional area of the surface.

$$
v_{\mathrm{C}}=0
$$

Regarding the flow to the outlet (indexed "TO"), B is a point on the surface of the control volume, A, where the pressure is assumed to be the static pressure asserted by the supernatant phase at rest at depth $t+H-h_{\mathrm{B}}$ as shown in Eq. (8).

$$
P_{\mathrm{B}, \mathrm{TO}}=P^{\circ}+t \rho_{2} g+\rho_{2} g\left(H-h_{\mathrm{B}}\right) .
$$

The height of point B can geometrically be determined from Fig. 2. In Eq. (9), the height is shown for spherical control volume, in Eq. (10) and in the height is shown for cylindrical control volume.

$$
\begin{array}{r}
h_{\mathrm{B}, \mathrm{TO}, \mathrm{s}}=H \sin \varphi ; \quad 0 \leq \varphi \leq \pi / 2 . \\
h_{\mathrm{B}, \mathrm{TO}, \mathrm{c}}=h ; \quad 0 \leq h \leq H \ldots \ldots . . .
\end{array}
$$

where index $\mathrm{s}$ and $\mathrm{c}$ corresponds to spherical and cylindrical control volume respectively while $\varphi$ and $\theta$ are defined in Fig. 2.

When calculating the flow through the outlet, B (indexed "TH") is a point on the outlet's cross-section area, $O$. Since the outlet area $O$ is in contact with the atmosphere, the pressure at $B_{\mathrm{TH}}$ can be approximated to equal the atmospheric pressure, as shown in Eq. (11) and the height, shown in Eq. (12), has been determined from Fig. 2.

$$
\begin{aligned}
& P_{\mathrm{B}, \mathrm{TH}}=P^{\circ} . \\
& h_{\mathrm{B}, \mathrm{TH}}=-l .
\end{aligned}
$$

The flow through the outlet can thus be calculated as follows.

$$
Q_{\mathrm{TH}}=\oint_{O} v d O
$$

Inserting Eqs. (5), (6), (7), (11), and (12) into Eq. (4) gives an expression for the velocity which can be inserted into Eq. (13) which gives the flow rate through the outlet shown in Eq. (14).

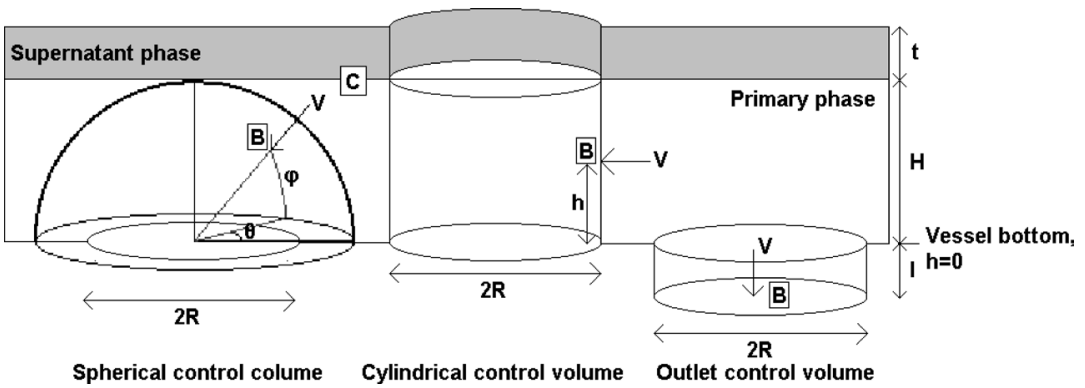

Fig. 2. Spherical control volume surrounding the outlet used in NMs, Cylindrical control volume surrounding the outlet used in NMc and the outlet control volume which balances the flow through the outlet in the NMs and NMc models. 


$$
Q_{\mathrm{TH}}=\pi R^{2} \sqrt{2 g\left(H+l+t \frac{\rho_{2}}{\rho_{1}}\right)}
$$

The flow through the surface area of the control volume and to the outlet can, for both spherical and cylindrical control volumes, be calculated as follows:

$$
Q_{\mathrm{TO}}=\oint_{A} v d A
$$

In the model with cylindrical control volume, the flow to the outlet can be calculated as seen below.

$$
Q_{\mathrm{TO}}=\int_{0}^{2 \pi} \int_{0}^{h} v d A
$$

Inserting Eqs. (5), (6), (7), (8) and (10) into Eq. (4) gives an expression for the velocity which can be inserted in Eq. (16) resulting in the flow rate expression shown in Eq. (17).

$$
Q_{\mathrm{TO}}=\int_{0}^{2 \pi} \int_{0}^{H} \sqrt{2 g\left(1-\frac{\rho_{2}}{\rho_{1}}\right)(H-h)} d h R d \theta
$$

After integration, the final flow rate expression for the cylindrical control volume is shown in Eq. (18).

$$
Q_{\mathrm{TO}}=\frac{4}{3} \pi R \sqrt{2 g\left(1-\frac{\rho_{2}}{\rho_{1}}\right)} \cdot H^{3 / 2}
$$

Hence, inserting Eq. (18) and Eq. (14) into the flow balance Eq. (3) gives equation NMc:

$$
R^{2}\left(H+l+T \frac{\rho_{2}}{\rho_{1}}\right)=\frac{16}{9} H^{3}\left(1-\frac{\rho_{2}}{\rho_{1}}\right)
$$

In the model with spherical control volume, Eq. (15) in combination with the geometry shown in Fig. 2, gives that the flow towards the outlet equals:

$$
Q_{\mathrm{TO}}=\int_{0}^{2 \pi} \int_{0}^{\pi / 2} v H^{2} \cos \varphi \cdot d \varphi \cdot d \theta
$$

Inserting Eqs. (5), (6), (7), (8) and (9) into Eq. (4) gives an expression for the velocity which can be inserted in Eq. (20) resulting in the flow rate expression shown in Eq. (21)

$$
Q_{\mathrm{TO}}=\int_{0}^{2 \pi} \int_{0}^{\pi / 2} H^{5 / 2} \cos \varphi \sqrt{2 g\left(1-\frac{\rho_{2}}{\rho_{1}}\right)(1-\sin \varphi)} d \varphi \cdot d \theta
$$

After integration Eq. (21) becomes the expression shown in Eq. (22):

$$
Q_{\mathrm{TO}}=\frac{4 \pi}{3} H^{5 / 2} \sqrt{2 g\left(1-\frac{\rho_{2}}{\rho_{1}}\right)}
$$

Hence, Inserting Eq. (22) and Eq. (14) into the flow balance Eq. (3) gives equation NMs:

$$
R^{4}\left(H+l+T \frac{\rho_{2}}{\rho_{1}}\right)=\frac{16}{9} H^{5}\left(1-\frac{\rho_{2}}{\rho_{1}}\right)
$$

The two new models are based on similar principles as previous models but calculate the volume flow to the outlet as an exact bath height dependent integral over the surface, and flow speeds determined in each point of the surface of the control volume as function of the second phase density and layer thickness.

\section{Results}

\subsection{Ideal Teeming}

Initially, the experimental teeming procedure in different vessels with different outlets was compared to a calculated teeming procedure. The predictions were done using the ideal Bernoulli equation (with no friction factor):

$$
P_{\mathrm{C}}+\rho g h_{\mathrm{C}}+\frac{\rho v_{\mathrm{C}}^{2}}{2}=P_{\mathrm{B}}+\rho g h_{\mathrm{B}}+\frac{\rho v_{\mathrm{B}}^{2}}{2}
$$

where $\mathrm{C}$ is a point on the bath surface and $\mathrm{B}$ is a point on the outlet cross-sectional area. Since the bath surface area is much larger than the outlet area, the velocity in $\mathrm{C}$ is assumed to be zero. The origin is placed on the vessel bottom. The $\mathrm{C}$ differential volume balance gives Eq. (25):

$$
v d t A_{\mathrm{OUT}}=A_{\mathrm{VESSEL}} d H \text {. }
$$

where $v$ is velocity in the outlet, $t$ is time, $d$ is differential operator, $A_{\text {OUT }}$ is outlet cross-sectional area, $A_{\text {VESSEL }}$ is vessel cross-sectional area and $H$ is bath height. The combination of Eq. (24) and Eq. (25) gives Eq. (26) which determines the total teeming time.

$$
t=-\frac{A_{\mathrm{VESSEL}}}{A_{\mathrm{OUT}}} \int_{H_{\mathrm{tot}}}^{0} \sqrt{2 g(H+l)} d H
$$

where $l$ is outlet length and $H_{\text {tot }}$ is initial bath surface height.

In Fig. 3, the bath level height is plotted versus the teeming time. The experimental and theoretical teeming curves align fairly well. However, a clear decrease in teeming rate can be seen at lower bath heights. This is caused by a combination of the onset of drain sink and the accumulated error from friction-factors in the system, especially the sharp edges surrounding the outlet.

Some experiments with varying initial filling heights were made in both the large and the small vessel and no dependence on drain sink formation height could be seen as long as the filling height was larger than the drain sink formation height. Thereafter, the results from the two vessels were compared with each other for similar outlet diameters and no significant difference could be found. Thus, the drain sink ought to be independent of vessel diameter as long as the vessel diameter is significantly larger than the outlet diameter. 


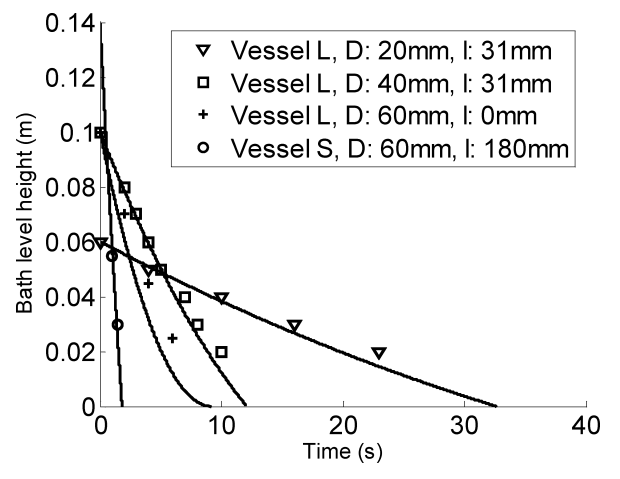

Fig. 3. Comparison between experimental data and ideal Bernoulli equation regarding bath level height as function of time for the experimental vessels, $D$ is diameter and $l$ is outlet length.

Trials were also made in the large vessel with the outlet positioned at different radial distances from the center of the bottom. No effect on drain sink formation height could be detected when the outlet was moved on the radius from the center of the vessel to a center to outlet distance of 0.65 times the vessel radius. Thus, the drain sink was found to be independent of initial filling height, vessel diameter and outlet position. All these results are in agreement with previous works. $^{1,3-8)}$

\subsection{Drain Sink Height}

In the results below, L\&S stands for Lubin and Springer model, ${ }^{11)} \mathrm{NMs}$ stands for the model with spherical control volume and NMc stands for the model with cylindrical control volume presented in Chap. 4 in the present paper.

In Fig. 4, the relationship between outlet diameter and drain sink height in the small vessel is shown for an outlet length of $0 \mathrm{~mm}$. The figure also includes the prediction by Andrzejewski model, ${ }^{1)}$ the relationship determined by Koria et al., ${ }^{5)}$ Lubin and Springer's hydrodynamic model ${ }^{11)}$ as well as the two new models developed in the present paper, NMc shown in Eq. (19) and NMs shown in Eq. (23). As seen, the NMc model and the model by Andrzejewski ${ }^{1)}$ show the best agreement with the experimental data. The predictions using the NMs model are also fairly close to the experimental data while the L\&S model predictions show less good accuracy.

The same experiments as shown in Fig. 4 were also performed in the large vessel. The experimental results together with the model predictions can be seen in Fig. 5 . Note that for the NMc and NMs models one line is for an experimental setup using an outlet length of $31 \mathrm{~mm}$. This is because when tapping the vessel through the $20 \mathrm{~mm}$ outlet, the tapping stream is cylindrical and sticks to the wall of the pipe through the vessel bottom of length $31 \mathrm{~mm}$, which is not the case for the larger outlets where the tapping stream has trumpet shape (free flow). However, the data for the LS-model and the model by Andrzejewski are only for an outlet length of $0 \mathrm{~cm}$. If the outlet length $31 \mathrm{~mm}$ is inserted into L\&S and Andzejewski models, L\&S overestimates the formation height even more than before and Andrzejewski model gives values in the range of the results from the NMc model.

In Fig. 6, the drain sink height as function of outlet length can be seen. There are two kinds of experimental

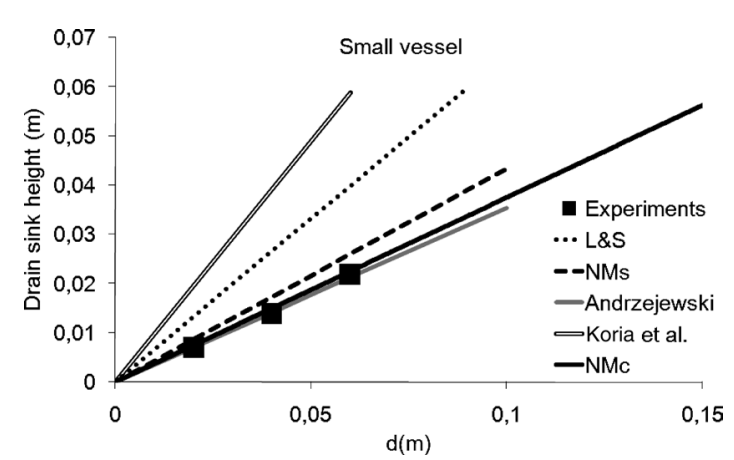

Fig. 4. Comparison between theoretical models and experimental data regarding drain sink formation height and outlet diameter in vessel $\mathrm{S}$.

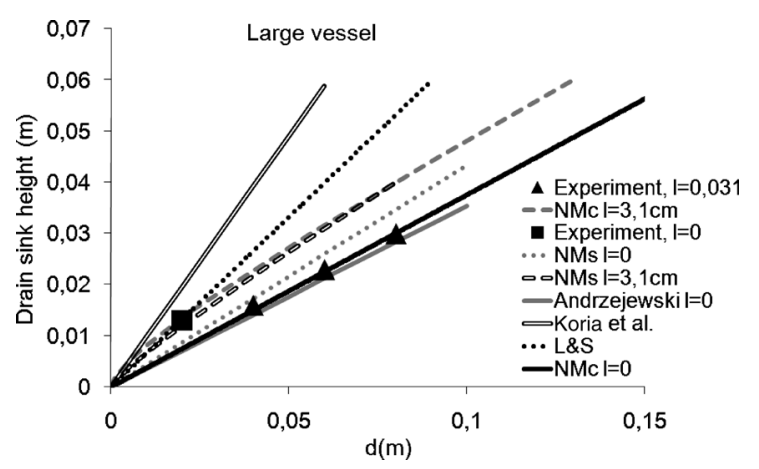

Fig. 5. Comparison between theoretical models and experimental data regarding drain sink formation height and outlet diameter in vessel $\mathrm{L}$.

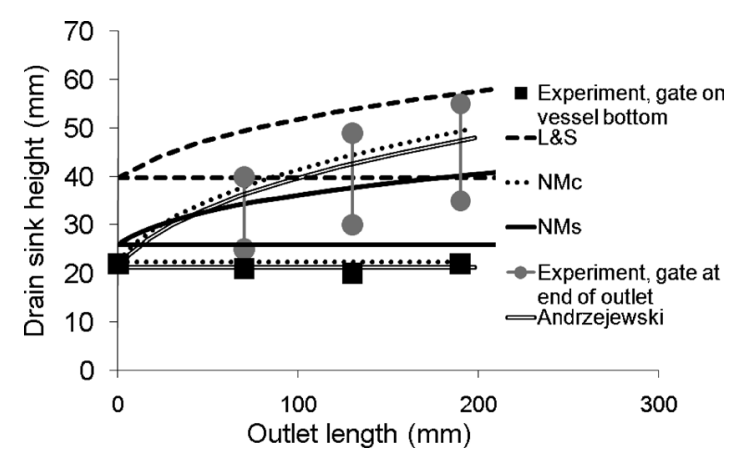

Fig. 6. Comparison between theoretical models and experimental data regarding drain sink formation height and outlet length for sliding gate positioned on vessel bottom and sliding gate positioned at the end of the constant-diameter outlet pipe, the experimental values reaches from drain sink dimple formation to drain sink penetration.

points for each outlet length. The black square shows the drain sink formation height when the sliding gate was situated on the vessel bottom and the gray circle shows the same when the sliding gate was placed at the end of the outlet pipe. When the sliding gate was placed on the vessel bottom, there was no water in the outlet pipe at tap start, the tapping stream was trumpet shaped and had no contact with the outlet pipe wall. When the sliding gate was placed at the end of the outlet pipe, the pipe was filled with water when the tapping began and during the teeming of the vessel, the tapping stream had constant contact with the outlet pipe wall. During these trials, the teeming flow rate was very high why the velocity of the bath surface became high. Thus, the surface moved a considerable distance during the 
drain sink formation why the experimental values shown in Fig. 6 reaches from drain sink dimple formation height to drain sink penetration height.

Trials were also carried out with other second phases than air. The effect of second phase density on drain sink height for $40 \mathrm{~mm}$ and $20 \mathrm{~mm}$ outlet diameter can be seen in Fig. 7 and Fig. 8 respectively. The figures also show the drain sink height values predicted by the L\&S and the NMs models. The second phase layer thickness was $10 \mathrm{~mm}$ or $20 \mathrm{~mm}$. The kinematic viscosity of the second phases are in order of increasing density $0.37 \mathrm{cSt}, 20 \mathrm{cSt}$ and $100 \mathrm{cSt}$ in Fig. 7 as well as $10 \mathrm{cSt}$ and $100 \mathrm{cSt}$ in Fig. 8.

\subsection{Shape of Vessel Bottom}

Figure 9 shows a comparison between flat and coned shaped bottom with respect to the amount of water left in the vessel when the second phase penetrates the outlet. The cone slope is 50 degrees $\left(0^{\circ}\right.$ corresponds to flat bottom and $90^{\circ}$ is vertical cylindrical pipe). The upper number beneath the horizontal axis in Fig. 9 is second phase viscosity in cSt

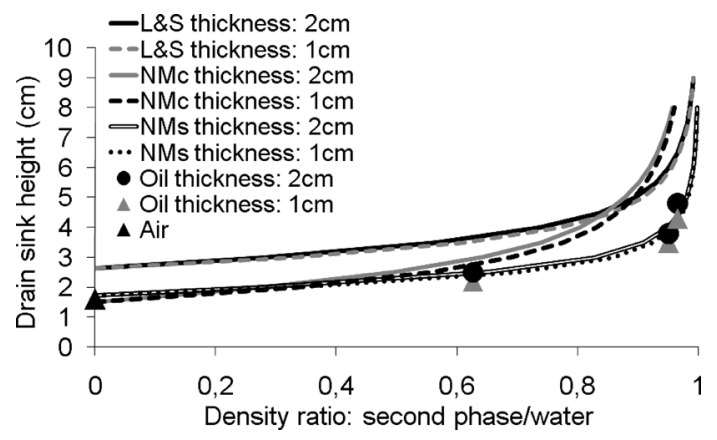

Fig. 7. Comparison between theoretical models and experimental data regarding drain sink formation height and density ratio of second phase and water, outlet diameter $40 \mathrm{~mm}$.

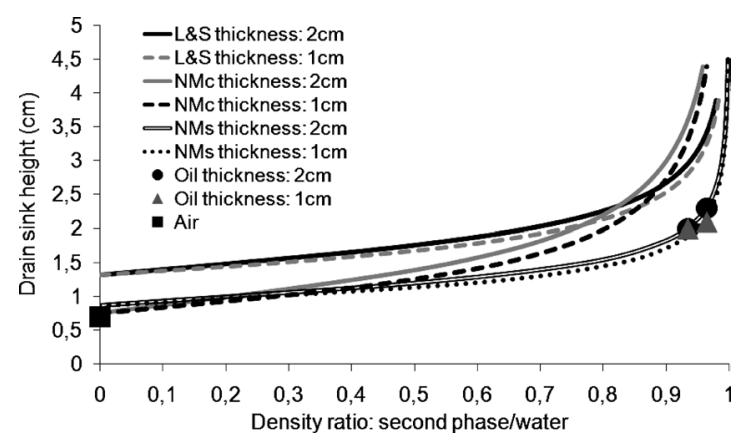

Fig. 8. Comparison between theoretical models and experimental data regarding drain sink formation height and density ratio of second phase and water, outlet diameter $20 \mathrm{~mm}$.

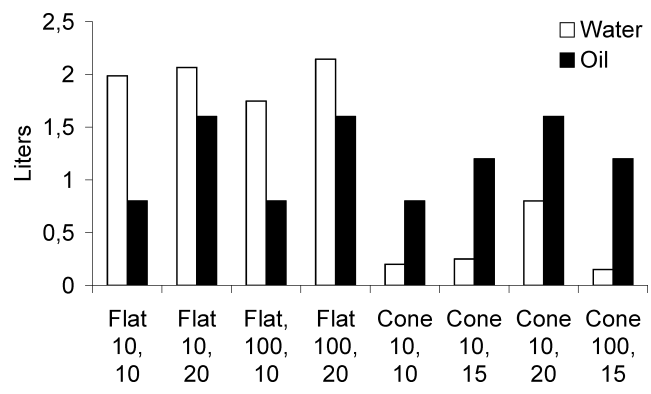

Fig. 9. Amount of second phase and water left in vessel $\mathrm{S}$ at onset of drain sink for flat and coned bottom shape. and the lower number is second phase layer thickness given in $\mathrm{mm}$. As can be seen, the amount of water left during the cone tapping is much less than the amount of second phase, while the opposite is valid for the flat bottom tapping.

\section{Discussion}

\subsection{Definition of Drain Sink and Drain Sink Forma- tion Height}

Before discussing the comparison between models and experimental data, it is important to have a clear definition of the drain sink and drain sink formation height. The drain sink is here defined as the funnel-shaped non-rotational two-phase boundary ranging from the vessel walls through the outlet. As for the definition of the formation height, Andrzejewski et al. ${ }^{1)}$ observed a hydraulic jump on the bath surface preceding the drain sink and considered it as an indication that the flow velocity had become critical. In the present experiments, the hydraulic jump was rarely observed and therefore not suitable for determining the drain sink formation height. In addition, the hydraulic jump was observed not to be directly related to the drain sink as it is defined above, but rather to be an effect of another change of the flow regime in the outlet. In Fig. 10, the cross-sections of four experimentally observed flow patterns are shown. Figure A represents a fully filled outlet flow with a low-pressure region in the upper part. The low-pressure region is probably a suction effect caused by low interfacial energy between water/wall and high interfacial energy between water/air. The velocity is constant over the outlet pipe, because the sum of the force due to the pressure gradient and the force due to gravity is zero. The flow pattern A was observed in outlets where the outlet length is much larger than the outlet diameter, here called narrow outlets. In addition, in outlets where the sliding gate was placed at the end of the outlet pipe instead of on the vessel bottom. If flow A does not occur, flow B will take place. In flow B, the pressure is close to the surrounding pressure in the whole tapping stream. Flow $\mathrm{C}$ is a drain sink occurring in an Atype of flow and Figure D shows a drain sink occurring in a B-type of flow. Sometimes, the flow pattern changes from an A-type flow to a B-type flow during tapping of the vessel. If this happens when the bath level is low, the rapid decrease of the tapping stream volume caused by the minimization of tapping stream surface area and the low pressure in the stream close to the vessel bottom, may cause a short-time back-flow of water observed as a hydraulic jump on the surface. The hydraulic jump can only be observed during change from A to B when no drain sink is present. Therefore, it is not a very accurate definition of the drain sink formation height. Thus, in this paper, the drain sink formation height is defined as the bath level corresponding to the onset of the surface dimple which grows and penetrates the outlet.

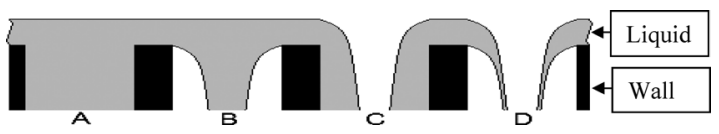

Fig. 10. Different teeming regimes, $\mathrm{A}$ is filled outlet pipe, $\mathrm{B}$ is non-filled outlet pipe, $\mathrm{C}$ is drain sink with filled outlet pipe and $\mathrm{D}$ is drain sink with non filled outlet pipe. 


\subsection{Drain Sink Height Results}

The results show that the drain sink height increases with an increased outlet diameter, outlet pipe length (if the flow fills the outlet pipe, see flow A in Fig. 10) and second phase density but is independent of second phase viscosity. Thus, the drain sink height for a certain outlet increases with increasing discharge rate, while it is not sensitive to second phase viscosity. This is probably because the flow velocity gradients in the second phase are low. As seen in the results, accurate prediction of the drain sink formation height could not be done with the Lubin and Springer model, although it previously has been reported to show good agreement with experimental results. ${ }^{1,3,11)}$ Better fit was obtained by the Andrzejewski model, but it does not consider effect of second phase. Furthermore, as the results of Andrzejew$\mathrm{ski}^{1}{ }^{1)}$ indicate, the predictions are worse when the drain sink height becomes high. Therefore, the two new models, NMc and NMs were developed.

The models might at first glance seem to be paradoxes because the critical height is defined as the bath height when the flow to the outlet at fully developed drain sink equals the flow trough the outlet at no drain sink. The key issue is the assumption regarding the pressure distribution at the outlet and on the surface of the control volume. When the second phase penetrates the primary phase, the pressure at the two phase interface approximately equals the pressure in the second phase at rest but still, the pressure at the outlet is atmospheric. Thus, until the second phase has fully penetrated the outlet, it might be reasonable to use the pressure distribution assumption described above, although the visual image of the assumption seems slightly awkward, especially for the case with spherical control volume.

As can be seen in the results, NMc together with Andrzejewski model show best results when the drain sink height is low compared to the outlet diameter while the NMs shows best agreement when the drain sink height is large compared to the outlet diameter. The L\&S model always overestimates the drain sink height in the present range of parameters while it had better fit than Andrzejewski model at very long outlet lengths, see Ref. 1). These results can be explained by the assumptions made in the models. The NMc and Andrzejewski models use a cylindrical control volume with a radius which equals that of the outlet thus assuming that the flow velocity to the outlet is purely horizontal. This is most likely to occur at bath levels less than, or close to, the outlet radius, why these models have best fit in systems where the drain sink forms at these heights. The NMs uses spherical control volume with a radius that is equal to the bath height, thus assuming velocities normal to a sphere surrounding the outlet. This model will overestimate the drain sink height at bath levels smaller than the outlet radius while it will be more correct when the drain sink occurs at higher bath levels, for example when a heavy second phase is present. The slight difference in results between NMc and Andrzejewski model lies in the calculation of the mean velocity to the outlet, where Andrzejewski defines the critical velocity as the velocity when the Froude number equals 1 while the NMc calculates the velocity using the Bernoulli equation, giving that the critical veloc- ity occurs when the Froude number equals 0.94 . The reason why the L\&S model which, similar to the NMs, uses spherical control volume, overestimates the drain sink height is probably that the flow velocity towards the outlet is assumed to be constant over the control volume and to equal the velocity given by the Bernoulli equation at the top of the sphere. Thus, the volume flow to the outlet becomes underestimated and the drain sink height becomes over estimated.

Based on the experimental results and predictions of the models, the lowest drain sink formation height can be obtained using short outlet lengths and low density second phases. However, even though the drain sink height is difficult to affect, the amount of steel left in the ladle at drain sink height can be controlled and minimized by the bottom design.

\subsection{Shape of Vessel Bottom}

Several authors has suggested inclined ladle bottom as a solution to the drain sink problem. Not because it will affect the drain sink height, but because an inclined bottom with an intelligent placement of the nozzle reduces the amount of steel left in the vessel when the drain sink forms. However, the inclined bottom is not fit for other measures against drain sink as for example partial closing of the sliding gate. Since part of the inclined bottom is lower then the nozzle opening, steel always will be left in the ladle. A conic shaped bottom would be better since bath height close to the outlet always is higher compared to a flat bottom. As seen in Fig. 9, the amount of slag carry-over was strongly reduced when the bottom was conic. The effect of cone shaped bottom compared to flat bottom is shown in Fig. 11 where the logarithmic of the ratio between the flow quotients to and through the outlet for the conic and flat bottom shapes are plotted over total amount of liquid in the vessel and radial position. The flow quotients plotted in the figure are defined by the expression shown in Eq. (27).

$$
\frac{Q_{\mathrm{ConeTo}} / Q_{\mathrm{ConeThrough}}}{Q_{\text {FlatTo }} / Q_{\text {FlatTrough }}}=\frac{Q_{\mathrm{ConeTo}} Q_{\text {FlatTrough }}}{Q_{\text {FlatTo }} Q_{\text {ConeThrough }}} \ldots . .
$$

where $Q_{i j}$ are calculated as:

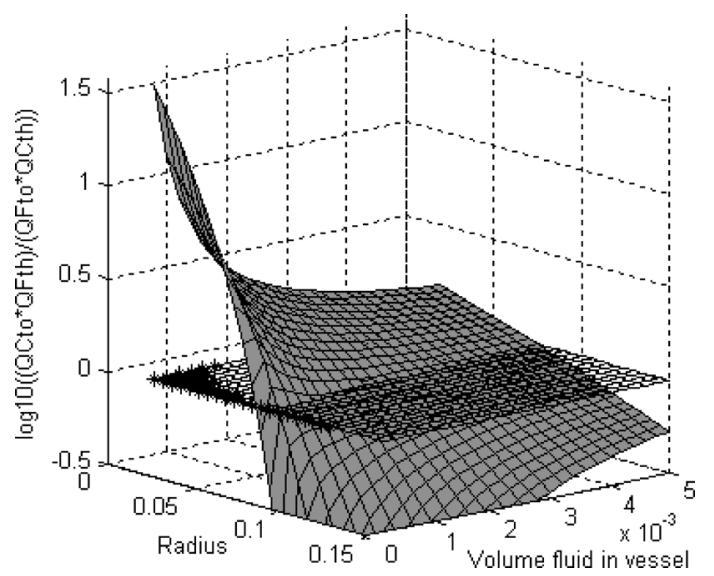

Fig. 11. Logarithmic ratio between volumetric flow rate quotient for coned and flat bottom shape, negative value means that the probability of drain sink formation is larger for the flat bottom than for the coned bottom vessel, positive value means the opposite. 


$$
\begin{gathered}
Q_{i \mathrm{To}}=2 \pi \cdot r \int_{0}^{H_{\mathrm{r}}} \sqrt{2 g h} d h \\
Q_{i \mathrm{Through}}=\pi \cdot R^{2} \sqrt{2 g H} \ldots
\end{gathered}
$$

where $r$ is distance between the point of interest and outlet, $H_{\mathrm{r}}$ is the liquid height at the radial position, $h$ is height, $g$ is acceleration due to gravity, $R$ is outlet radius and $H$ is total liquid height above the outlet. The properties of the logarithmic flow quotient shown in Eq. (27) are that it takes a value larger than zero if the drain sink is more likely to form in the flat bottom system rather than in the cone bottom system and it takes a value less than zero if the drain sink is more likely to form in the cone bottom system than in the flat bottom system. The black stars in Fig. 11 mark the domain where the flow, according to NMc, becomes critical for the flat bottom. As clearly can be seen the conic bottom shape is more than $1000 \%$ better than the flat bottom at short radius and low amount of liquid left while the flat bottom shape is better at large radius. The latter is not important since it is an effect of the infinite bath level height at maximum radius when the volume of liquid is less than the volume of the cone. Since the critical transport region is through the outlet, local phenomena at large radius far from the outlet is not of importance. Note that these calculations assume center placed outlet and that the results will be quantitatively but not qualitatively different if applied to an off-center placed outlet. The latter is true since the flow through the outlet as seen in Eq. (29) only increases with the square root of the bath height while the flow to the outlet increases with the exponent 1.5 of the bath height as seen if integrating Eq. (28). It is also important to note that in an industrial ladle with, for example, a 2 meter radius, every centimeter of increased yield during tapping corresponds to almost $1000 \mathrm{~kg}$ extra steel teemed from the ladle. Thus, a strong economical driving force to decrease the negative impact of the drain sink exists.

\section{Conclusions}

The behavior of an unsteady-state drain sink as function of outlet diameter, outlet length, second phase viscosity, second phase density and second phase layer thickness was studied using physical modeling. The drain sink formation height was found to increase with an increased outlet diameter, outlet pipe length, and second phase density while it was found to be independent of a change of viscosity of the second phase. In addition to the experiments, two new hydrodynamic models to predict the drain sink formation height were derived based on the ideal Bernoulli equation. Predictions using these models were found to agree well with experimental drain sink height data. Moreover, the new models were found to agree better with the current experimental data in comparison to predictions using other models presented in the open literature. Furthermore, both new models consider the effect of second phase on the drain sink formation height predictions, which the previous cylindrical model had not taken into account. It is also discussed in which situations different kinds of models are suitable. Finally, it was discussed that the geometry of the vessel bottom will influence the yield in industrial steel practice. Here, it was found that cone shaped bottoms are preferred in comparison to flat vessel bottoms.

\author{
Nomenclature \\ $R$ : $\quad$ Outlet radius (m) \\ $r:$ Radial position $(\mathrm{m})$ \\ $S:$ Constant $\left(\mathrm{m}^{0.5} \mathrm{~s}^{-1}\right)$ \\ $H$ : Surface height $(\mathrm{m})$ \\ $T:$ Constant $\left(\mathrm{m}^{0.5} \mathrm{~s}^{-1}\right)$ \\ $U:$ Constant (-) \\ $H_{\mathrm{cr}}$ : Critical surface height (m) \\ $Q_{\mathrm{cr}}$ : Critical flow rate $\left(\mathrm{m}^{3} \mathrm{~s}^{-1}\right)$ \\ $\rho$ : Density $\left(\mathrm{kg} \mathrm{m}^{-3}\right)$ \\ $g$ : Gravitational acceleration $\left(\mathrm{m} \mathrm{s}^{-2}\right)$ \\ $t$ : Supernatant phase thickness (m) \\ $A$ : Outlet cross-sectional area $\left(\mathrm{m}^{2}\right)$ \\ $h$ : Vertical distance $(\mathrm{m})$ \\ $v:$ Velocity $\left(\mathrm{m} \mathrm{s}^{-1}\right)$ \\ $P$ : $\quad$ Pressure $(\mathrm{Pa})$ \\ $A$ : Surface area of control volume $\left(\mathrm{m}^{2}\right)$ \\ $O:$ Surface area of outlet $\left(\mathrm{m}^{2}\right)$ \\ $\varphi$ : Vertical angle (rad) \\ $\theta$ : Horizontal angle (rad)
}

\section{REFERENCES}

1) P. Andrzejewski, A. Diener and W. Pluschkell: Steel Res., 12 (1987), 547.

2) A. V. Kuklev, V. V. Tinyakov, Yu. M. Aizin, V. N. Gushchin and V. A. Ulíyanov: Metallurgist, 5-48 (2004), 207.

3) R. Sankaranarayanan and R. I. L. Guthrie: Ironmaking Steelmaking, 29 (2002), 147.

4) G. Mazzaferro, M. Piva, S. Ferro, P. Bissio, M. Iglesias, A. Calvo and M. Goldscmit: Ironmaking Steelmaking, 31 (2004), 503.

5) S. Koria and U. Kanth: Steel Research, 1 (1994), 8.

6) M. Dubke and K. Schwerdtfeger: Ironmaking Steelmaking, 17 (1990), 184.

7) P. Hammerschmid, K. H. Tacke, H. Popper, L. Weber, M. Dubke and K. Schwerdtfeger: Ironmaking Steelmaking, 11 (1984), 332.

8) R. Sankaranarayanan and R. Guthrie: Proc. to Int. Symp. on Developments in Ladle Steelmaking and Continuous casting, CIM, Hamilton, (1990), 66.

9) M. Nadif, J. Lehman, M. Burty and J. F. Domgin: Proc. the 7th Int. Conf. on Clean Steel, OMBKE, Balatonfüred, (2007), 38.

10) L. Brinkmeyer and S. D. Melville: Ironmaking Steelmaking, 22 (1995), 45

11) B. Lubin and G. Springer: J. Fluid Mech., 29 (1967), 385.

12) S. Feldbauer and A. W. Cramb: 13th PTD Conf. Proc., ISS, Warrendale, PA, (1995), 327.

13) J. A. Fay: Introduction to Fluid Mechanics, MIT Press, Warrendale, TMS, (1994), 17.

14) D. R. Poirier and G. H. Geiger: Transport Phenomena in Materials Processing, The Minerals, Metals \& Materials Society, Warrendale, PA, (1994), 21.

15) J. Szekely and N. Themelis: Rate Phenomena in Process Metallurgy, John Wiley \& Sons, New York, (1971), 10.

16) D. Gupta and A. K. Lahiri: Metall. Mater. Trans., 27 (1996), 695.

17) Data from http://www.shinetsu.co.jp/e/index.shtml.

18) Data from www.vwr.com.

19) Data from http://www.junsei.co.jp/.

20) M. Iguchi, K. Takahashi, O. Ilegbusi, M. Sano and A. Kiushi: ISIJ Int., 38 (1998), 1032

21) F. M. White: Fluid Mechanics, Second Edition, McGraw-Hill, New York, (1979), 690. 